Slavica

bruxellensia

\section{Slavica bruxellensia}

Revue polyphonique de littérature, culture et histoire

slaves

$6 \mid 2010$

Linguistique russe

\title{
De la psycholinguistique slave au folklore ukrainien
}

Introduction à la pensée d'Aleksandr A. Potebnja

Daniel S. Larangé

\section{OpenEdition}

Journals

Édition électronique

URL : http://journals.openedition.org/slavica/344

DOI : $10.4000 /$ slavica.344

ISSN : 2034-6395

Éditeur

Université libre de Bruxelles - ULB

Édition imprimée

Pagination : 19-30

ISSN : 2031-7654

Référence électronique

Daniel S. Larangé, «De la psycholinguistique slave au folklore ukrainien », Slavica bruxellensia [En ligne], 6 | 2010, mis en ligne le 15 juin 2010, consulté le 19 avril 2019. URL : http://journals.openedition.org/ slavica/344; DOI : 10.4000/slavica.344

Ce document a été généré automatiquement le 19 avril 2019.

\section{cc) $(9)$}

Les contenus de Slavica bruxellensia sont mis à disposition selon les termes de la Licence Creative Commons Attribution - Pas d'Utilisation Commerciale - Pas de Modification 3.0 France. 


\title{
De la psycholinguistique slave au folklore ukrainien
}

\author{
Introduction à la pensée d'Aleksandr A. Potebnja
}

Daniel S. Larangé

1 Aleksandr A. Potebnja (1835-1891) est redécouvert en Russie comme un important philosophe ${ }^{1}$ grâce à de récentes rééditions; en Ukraine, il est devenu comme un des premiers folkloristes ${ }^{2}$ et pères de la nation, aux côtés de Taras Ševčenko (1814-1861) et Ivan Franko (1856-1916)3. Pourtant, il s'inscrit dans la tradition de la linguistique allemande, de Friedrich Wilhelm von Humboldt (1767-1835), se référant explicitement à Gotthold Ephraim Lessing (1729-1781) et Johann Wolfgang von Goethe (1742-1832) qui manifestaient un grand intérêt pour les formes brèves et les traditions régionalistes.

2 Quel rapport s'établit-il, dans la linguistique, entre l'étude descriptive de la langue (morphologie, lexique et syntaxe) et le travail de classification et de typologie des éléments du folklore ukrainien, rassemblant les dictons, les anecdotes, les dialectes, les chants, les danses et la broderie ? À partir de cette question, une seconde peut être formulée : Pourquoi Potebnja est-il célébré à la fois comme un héraut de l'identité ukrainienne et un philosophe russe?

3 Un lien étroit unit ces deux interrogations. Le folklore, la langue et l'identité nationale sont imbriqués de sorte que l'étude de l'un de ces composants nécessite une mise en perspective des deux autres. Une brève présentation de la vie et de l'œuvre de Potebnja montre que la philosophie de la langue inscrit la description ethnographique dans des considérations linguistiques. Cette manière de procéder permet alors de distinguer une véritable identité ukrainienne fondatrice au cœur des peuples slaves.

\section{Aleksandr Afanas'evič Potebnja (1835-1891)}

4 Potebnja naît le 22 octobre 1835 dans le village de Havrilovka, dans la région de Poltava, en Ukraine. Il quitte en 1851 le gymnase polonais de Radom, où il a suivi la classe 
d'allemand et de latin, pour entrer à la faculté de droit de l'Université de Kharkiv. L'année suivante son ami Mikhail Vasi’evič Negovskij, l'un des premiers compilateurs des chansons populaires ukrainiennes, le convainc de s'inscrire à la Faculté d'histoire et de philologie. Il y subit les influences d'abord des jumeaux Pëtr Alekseevič Lavrovskij (1827-1886), slaviste et philologue, et Nicolaj Alekseevič Lavrovskij (1827-1899), grammairien et théoricien de la littérature, qui le sensibilisent à l'identité ukrainienne par l'étude de l'onomastique ${ }^{4}$, et ensuite du professeur Amvrosij Luk'janovič Metlinskij (1814-1870), amateur du folklore petit-russe ${ }^{5}$. Il obtient en 1856 le grade de kandidat en soutenant un mémoire intitulé : Pervye gody vojny ChmeL'nickogo (Les Premières Années de guerre de Khmielnicki). Le choix de son sujet témoigne déjà de son engagement politique : son frère Andrej Afanas'evič Potebnja (1838-1863), membre actif de la société secrète révolutionnaire Zemlja $i$ volja(Terre et volonté), est tué lors de l'insurrection polonaise de 1863. Il a été célébré par les dirigeants, Aleksandr Ivanovič Herzen (1812-1870) et Nikolaj Platonovič Ogarëv (1813-1877), comme « un martyr national »6.

5 Potebnja enseigne la langue et la littérature au gymnase $n^{\circ} 1$ de Kharkiv jusqu'en 1860. Un an plus tard, il présente un mémoire de maîtrise en littérature comparée $O$ nekotorykh simvolakh $v$ slavjanskoj narodnoj poezii (De quelques symboles dans la poésie nationale) qui lui permet d'obtenir un poste d'assistant (adjunkt) au Département de langue et de littérature russes de l'Université de Kharkiv. Il y démontre la continuité entre le chant populaire et la fable orale d'une part et la poésie écrite d'autre part. La littérature serait l'expression culturelle d'une tendance naturelle à l'espèce humaine ${ }^{7}$. D'où la nécessité de normaliser la littérature ukrainienne afin de définir son identité ${ }^{8}$.

Il est alors envoyé en stage de recherche, de 1861 à 1862, à l'étranger. Il étudie le sanskrit à Berlin sous la direction de l'indianiste et historien Albrecht Weber (1825-1901), le tchèque à Prague, puis le serbo-croate et le slovène à Vienne. Il en profite pour publier une série d'articles sur la théorie de la littérature. Ces textes sont rassemblés sous le titre Mys' i jazyk (La Pensée et la langue). La mort de son frère l'oblige à revenir en Ukraine et à reprendre son enseignement à l'Université de Kharkiv en qualité de conférencier.

7 En 1865 , le jury de doctorat rejette la thèse qu'il consacre à la signification mythologique des rituels et superstitions: O mifičeskom značenii nekotorykh poverij $i$ obrjadov (De la signification mythique de quelques croyances populaires et rites). Suite à cela, il publie principalement des comptes-rendus sur ses travaux de phonologie et de dialectologie des Slaves orientaux. Il soutient en 1874 une nouvelle thèse de doctorat: Iz zapisok po russkoj grammatike (Des notes sur la grammaire russe). Derrière ce titre peu engageant, se trouve la première grammaire raisonnée de langue russe traitée d'un point de vue encyclopédique. En se basant sur un corpus puisé dans toutes les langues slaves et baltoslaves, les dialectes et le folklore, il dresse les fondements d'une morphologie et d'une syntaxe comparées. Son succès est tel qu'il est élu, en 1877, membre de la Société des amateurs de la langue et de la littérature russe de l'Université de Moscou et correspondant de l'Académie des Sciences. Le premier tome de sa thèse reçoit alors le fameux prix Lomonosov de l'Académie des Sciences. En 1891,la Société géographique russe lui attribue son grand prix, la Médaille de Constantin, pour ses mérites dans le domaine de l'ethnographie. Il meurt peu après, le 29 novembre, à Kharkiv.

De nombreux linguistes et théoriciens de la littérature ont suivi l'enseignement de Potebnja, tels Dmitrij Nikolaevič Ovsjanniko-Kulikovskij (1853-1920)' ${ }^{9}$, Aleksandr VasiL’evič Popov (1855-1880), Vasilij Ivanovič Kharciev (1865-1937) ${ }^{10}$, Aleksej VasiL’evič Vetukhov $(1868-1946)^{11}$ et Boris Andreevič Lezin $(1880-1942)^{12}$. 
9 Ses thèmes de prédilection concernent l'origine et l'évolution de la langue, les liens entre la langue et la pensée, les langues et les nations, le folklore et le mythe, le symbolisme de la langue, le folklore et l'art. Sa conception linguistique est foncièrement philosophique et se résumerait ainsi : la langue est le produit naturel de la société (d'où le mot ne saurait se réduire au seul produit de la conscience humaine), mais s'inscrirait dans une histoire d'échanges ${ }^{13}$.

\section{Une philosophie des langues}

Potebnja ouvre à la linguistique descriptive deux voies interprétatives, multipliant entre elles les ponts et passages. En effet, la psycholinguistique et la sociolinguistique sont beaucoup plus complémentaires qu'on ne le pense encore aujourd'hui. Puisque la langue est conçue comme le lieu de l'humanisation, l'âme individuelle et l'esprit collectif s'y révèlent. C'est pourquoi l'étude des énoncés et de leurs articulations permet de tenir un métadiscours qui concerne en priorité l'anthropologie. Il prend en considération le contexte et les conditions d'énonciation ${ }^{14}$, notamment l'intonation, le phrasé, la disposition du corps (les gestes et grimaces). Cela le conduit à distinguer la langue orale de la langue écrite dans la mesure où elles forment des médias différenciés. Néanmoins la langue orale dépend de sa variante écrite et les structures qui la charpentent partagent des similitudes fondamentales.

11 Par ailleurs, les résultats de la philologie méritent d'être intégrés à la description linguistique. Potebnja insiste sur l'historicité des langues qui détermine leur identité et oriente leur développement. La langue constitue la mémoire d'une communauté sociale et religieuse. Les représentations du monde (die Weltanschauungen) dépendent de l'histoire des langues, tant du point de vue de la construction des concepts et notions par la définition des dénotations que par leur articulation à travers leurs connotations et la construction syntaxique. Chaque communauté linguistique cultive un imaginaire qui lui est propre et qui ne se comprend qu'au regard de son histoire et de l'histoire de son idiome $\mathrm{e}^{15}$. Toute une gradation se dessine alors en partant de la langue, en passant par la culture et en aboutissant à la civilisation ${ }^{16}$. Chaque niveau se complexifie en intégrant l'ensemble des structures sous-jacentes.

12 En effet, la langue est définie comme un système général et généralisé, au point que c'est l'univers tout entier qui «prend sens " pour nos yeux et nos oreilles. Autrement dit, l'analyse locale est significative pour une lecture globale et l'étude générale sert aux interprétations plus circonscrites. En ce sens, John Fizer a raison de considérer Potebnja comme l'un des précurseurs du structuralisme : il y aurait une analogie formelle entre les différentes structures et entre superstructures et infrastructures ${ }^{17}$.

Cette conception globale et psychosociale du phénomène linguistique conduit Potebnja à œuvrer pour une reconstruction de la langue nationale. Comme pour Humboldt, la langue produit la pensée humaine : les catégories grammaticales sont des catégories cognitives ${ }^{18}$, autrement dit des formes de mouvement de la pensée ${ }^{19}$. Elle n'exprime pas « une pensée toute faite " mais elle sert d'instrument à une réflexion personnelle et créative. C'est par et à travers elle que nous percevons et appréhendons un monde reconstruit. Telle une vitre, elle filtre la lumière et réfléchit les images extérieures déformées par notre propre reflet. Le mot crée de la sorte un mythe, et le folklore, comme la science, ne forme qu'un discours parmi d'autres. C'est précisément dans le mot que l'individu peut objectiver sa 
perception du monde. L'histoire de la grammaire est donc l'histoire des types de pensée et de conscience.

Patrick Sériot et Tatjana Zarubina du Centre de recherches en histoire et épistémologie comparée de la linguistique d'Europe centrale et orientale de l'université de Lausanne distinguent deux périodes dans la vie scientifique de Potebnja :

1) Les années 1860-1865 sont consacrées à l'élaboration d'une théorie philosophique et psychologique de la langue. Il s'agit alors de poursuivre dans la voie déjà engagée par les idées de Humboldt ${ }^{20}$, de Chaïm Heymann Steinthal (1823-1899) ${ }^{21}$ et de Johann Friedrich Herbart (1776-1841) ${ }^{22}$ qui estiment que les éléments de base du psychisme (chez Herbart il s'agit des représentations) expliquent l'Esprit du peuple, selon l'expression hégélienne ${ }^{23}$, tel qu'il se reflète dans la langue, les mythes, les coutumes, la cuisine, le tissage..., autrement dit, dans tout phénomène culturel.

Chaque peuple crée sa propre langue en tant « qu'un seul penseur, un seul philosophe $»^{24}$. Les usagers perçoivent la réalité à travers le prisme des formes internes, imprégnées dans l'idiome. D'où le principe selon lequel les sociétés forment leur(s) langue(s) et registre(s) comme les langues configurent leur propre espace linguistique et construisent leur société. Il appert alors que l'art relève à la fois d'une création personnelle et d'une production sociale: il reflète l'image d'une époque perçue à travers une conscience historique ${ }^{25}$.

Dans MysL' i jazyk, il soutient que l'individu assimile les structures mentales de son milieu grâce à l'acquisition de la langue. Dès lors, toute pensée s'avère impossible hors du langage. Celle-ci naît sous une forme verbale : « Le mot naît en l'homme involontairement et instinctivement $»^{26}$. Le langage est non seulement la condition obligatoire de l'apparition de la pensée mais également son principal mode de développement.

C'est pourquoi Potebnja est aujourd'hui considéré comme l'initiateur d'une psychologie historico-culturelle ${ }^{27}$. La langue nationale, langue maternelle dans laquelle tout individu a grandi et a développé ses émotions et ses conceptions, est l'organe créateur de la pensée ${ }^{28}$ . L'esprit d'un peuple est alors appréhendable dans sa particularité nationale, à partir des témoignages littéraires et folkloriques, reflétés dans la pratique quotidienne et littéraire de la langue. Il recommande même le bilinguisme, voire le plurilinguisme, afin de développer davantage les aptitudes intellectuelles des enfants ${ }^{29}$. Il n'en demeure pas moins qu'en ce qui concerne les petits-Russes, leurs langues naturelles, qui remontent à l'origine du paléoslave et de la Rus kiévienne, restent l'ukrainien ou le biélorusse, et non le russe imposé par l'impérialisme tsariste.

19 2) La deuxième période de l'activité scientifique (1865-1891)de Potebnja est davantage consacrée à la dialectologie, la grammaire et la phonétique du russe et d'autres langues slaves, s'appuyant sur les données de la linguistique indo-européenne. Il s'agit de démontrer l'apport riche et varié des autres peuples slaves, notamment des Ukrainiens et des Biélorusses, au progrès du russe, comme c'est le cas dans sa monographie de 1870, Zametki o malorusskom narečii (Remarques sur le dialecte petit-russe).

Le romantisme allemand a fortement influencé différentes théories philosophiques et linguistiques en Russie, notamment le nationalisme ${ }^{30}$. L'idée hégélienne du développement (die Entwicklung) et la thèse de Friedrich Wilhelm Joseph von Schelling (1775-1854) de la valeur en soi (die Eigenwert) de la culture nationale ont permis de fonder une philosophie linguistique originale (samobytnaja lingvofilosofija, une philosophie 
originale de langue) par les penseurs slavophiles déjà sensibles au formalisme et à la psychologie des masses.

Aleksej Stepanovič Khomjakov (1804-1860), Konstantin Sergejovič Aksakov (1817-1860) et Potebnja ont développé une sorte de phénoménologie de l'esprit russe, objectivée dans la langue et établie sur l'idéalisme allemand. Potebnja a été particulièrement influencé par Schelling, Emmanuel Kant (1724-1804) ${ }^{31}$ et Georg Wilhelm Friedrich Hegel (1770-1831) : l'homme prend conscience de soi uniquement dans le langage ( $v$ slove $)^{32}$.

Potebnja leur emprunte l'idée selon laquelle les sensations subjectives interviennent considérablement dans la perception et la connaissance du monde ${ }^{33}$. De plus, il récupère la thèse de Humboldt sur l'objectivation de la langue effectuée dans les relations de l'homme à une nature qui agit immédiatement dans la "transmission» des représentations subjectives ${ }^{34}$. Il insiste sur l'interaction entre la société et l'individu qui se répercute dans le développement des langues. L'écrivain transforme ainsi à chacune de ses performances la langue qu'il a reçue en héritage et lègue à la postérité une langue renouvelée. Cela conduit le psycholinguiste à envisager alors un subtil travail dialectique entre la pratique individuelle de la langue et l'inscription de tout énoncé dans une communauté de réception. Il s'agit donc bien d'étudier les processus cognitifs mis en œuvre dans le traitement et la production du langage ${ }^{35}$.

\section{La forme interne de la langue}

Une des notions que travaillent Humboldt et Potebnja est celle de la « forme interne ». Si Humboldt parle de «forme interne de la langue »(innere Sprachform) $)^{36}$, Potebnja défend davantage le concept d'une «forme interne du mot » qui est un rapport entre le contenu de la pensée et la conscience : La forme interne du mot est un rapport du contenu de la pensée à la conscience ; elle montre comment l'homme se représente sa propre pensée. » ${ }^{37}$ Selon Humboldt, la langue est conçue comme un système total où même le moindre élément ne peut apparaître sans être lié au principe unique de la forme, traversant toutes les parties de la langue. Cette forme interne est constituée par ce qui reste constant et uniforme dans l'activité de l'esprit, à savoir cette essence qui hausse le son articulé jusqu'à l'expression de la pensée. Grâce à cette forme interne, la langue peut reconstruire sa spécificité, et chaque idiome véhicule en lui une manière de vivre et de sentir différemment, autrement dit toute une axiologie partagée par une nation, par une communauté ou par une famille ${ }^{38}$.

La définition de la forme interne de la langue n'a pourtant jamais été suffisamment clarifiée par Humboldt, ce qui engendre une confusion dans la conception de la nature des relations entre la langue et la pensée. La notion elle-même sert de «base conciliatoire » pour expliquer l'harmonie entre la pensée et la langue, tout comme René Descartes recourt à l'idée de Dieu pour expliquer le lien entre les deux substances antagonistes, le corps et l'âme.

Elena Kokoškina dans un article consacré à l'évolution de la notion d'innere Sprachform donne trois définitions de la forme interne chez Humboldt ${ }^{39}$ :

26 1) elle a une fonction fédératrice en assurant la connexion entre le concept et le son, c'est-à-dire entre les catégories de la pensée et les formes matérielles de la langue ;

2) elle justifie l'expression « d'esprit du peuple », qui se réalise dans la langue ;

3) elle désigne la structure même de la langue, comprise abstraitement. 
27 À côté de sa fonction régulatrice en linguistique, la «forme interne » correspond chez Humboldt à une affirmation accrue de l'unité et de l'autonomie de chacune des langues. Une langue n'est pas l'émanation d'une nation donnée; c'est un être et non plus un simple phénomène.

28 Cela ne veut pas dire que les langues auront toutes la même valeur spirituelle, ni que l'individu sera prisonnier de la langue ; mais, dans son chemin vers la reconnaissance de cet être de la langue, Humboldt prend le risque de rouvrir le dualisme qu'il voulait éviter, en l'occurrence quand la « forme interne de la langue » évoque quelque sujet spirituel qui se servirait des outils grammaticaux et lexicaux ${ }^{40}$.

29 Le terme de "forme interne de la langue " ne saurait se résumer à une définition univoque. Ce fait est à l'origine des différentes interprétations de cette notion proposées par les partisans de Humboldt et les historiens de la linguistique.

Inspiré par Humboldt, Potebnja crée sa propre conception de la langue qu'il présente dans Mys' i jazyk. Pour résoudre les problèmes du rapport entre la pensée et la langue et étudier le rôle de la langue dans la connaissance, il élabore sa propre interprétation et fait de la notion de «forme interne » une notion plus particulière et concrète, une notion de «forme interne du mot ».

31 Le mot est une synthèse de trois éléments : la forme externe, c'est-à-dire, le son articulé ( členorazdeL'nyj), le contenu (soderžanie), qui est objectivé (ob'ektivnoe) par le son, et la forme interne, le moyen par lequel est exprimé le contenu ${ }^{41}$. Potebnja éclaircit ainsi la différence entre le contenu et la forme interne :

La « forme interne », mise à part l'unité réelle de la représentation, permet également la connaissance de cette unité. Citant Humboldt, Potebnja explique que l'homme essaie d'unir les objets qui, par la multiplicité de leurs représentations, agissent sur lui. Pour exprimer cette unité, l'homme a recours à une entité phonique, celle du mot. Le son n'évince aucune des représentations produites par l'objet, mais devient leur récipient. Potebnja accorde donc plusieurs acceptions au terme « forme interne » :

33 1) Au sens étymologique (le plus proche [bližajšee]) du mot, elle témoigne du lien de causalité entre le sens primitif et le sens actuel et subjectif du mot.

2) Elle désigne la relation du contenu de la pensée envers la conscience, et montre «comment l'homme se représente sa propre pensée ».

3) Elle signifie le trait de la représentation qui domine les autres, c'est-à-dire, le centre de la représentation.

4) La forme interne du mot peut être comprise non pas comme l'image de l'objet, mais comme l'image de l'image (obraz obraza) ${ }^{43}$.

Potebnja définit la forme interne du mot comme « un rapport du contenu de la pensée à la conscience ; elle démontre comment l'homme représente sa propre pensée ${ }^{44}$. Le mot possède une épaisseur qui correspond à un vécu en société. Il renvoie à une multitude d'expériences partagées par une communauté linguistique et culturelle. À ce titre, il transporte et provoque une émotion actualisée par son usage régulier dans des situations précises.

Cette définition contient toutefois quelques contradictions. On peut accepter que la forme interne du mot soit un rapport de la pensée à la conscience, si l'on considère la pensée comme extérieure et objective à la conscience. Cependant, le linguiste ne le pense pas, car il sous-entend par "forme interne » la représentation de la pensée propre à l'homme dans la conscience. Ce qui signifie qu'il existe en l'homme une sphère de pensées 
inconscientes pourtant accessibles par la forme interne du mot. Cette sphère inconsciente est même désignée comme étant l'âme. Mais si la pensée et sa prise en conscience sont subjectives, comment peut émerger, dans ce cas, une forme interne du mot à la fois objective et langagière?

En revanche, une pareille définition de la forme interne du mot entraîne le fait que la pensée inconsciente et sa prise en conscience précèdent le mot, que le mot avec sa forme interne est le résultat de l'activité de la pensée et puis de la conscience. Dans un autre passage, Potebnja reconnaît la primauté du mot sur la pensée sous la forme d'une image sensorielle (čuvstvennyj obraz) ou sous la forme de notion (ponimanie). Par conséquent, le mot devient à la fois un moyen et le résultat d'une activité.

Ces contradictions sont aux fondements ontologiques et épistémologiques d'une théorie qui prétend que c'est une illusion de penser que «nous voyons, percevons etc. les objets eux-mêmes et non nos propres impressions $»^{45}$. En cela, elle partage la thèse selon laquelle le monde est «ma » représentation, une représentation intersubjective. Cette position manquerait néanmoins de cohérence. D'abord elle exclut le fait que d'autres gens reconnaissent leur réelle existence (ce qui est incompatible avec le processus de la langue et de la communication), ensuite il est question des objets du monde externe en tant qu'existant, dans la mesure où « leur forme interne ne désigne plus un sentiment mais un trait objectif de l'objet ». Une telle position ontologique s'appuie sur une épistémologie sensualiste qui admet comme évidente la thèse aristotélicienne puis thomiste selon laquelle " en terme de connaissance, on sait que nihil est in intellectu, quod non prius fuerit in sensu » (Rien n'est dans l'intellect qui ne soit d'abord passé dans les sens). Il reconnaît d'ailleurs que, sans aucune intention de sa part, l'homme perçoit les sons de sa propre voix.

La notion de «forme interne du mot » occupe une place importante. Potebnja admet qu'il faudrait rechercher le lieu commun de toutes les langues, leurs ressemblances qui prouvent l'origine des peuples qui les parlent, et celles qui relèvent de l'origine de l'humanité. Cette tâche lui semble trop ambitieuse. En effet, on devrait : 1) répertorier les formes externes et internes primitives de tous les mots ; 2) décrire la forme interne de chaque mot ; 3) définir les qualités des premiers sons.

Les conceptions de la «forme interne de la langue » (innere Sprachform) ${ }^{46}$ et de la « forme interne du mot » (innere Wortform) ont inspiré en Russie les recherches sur «l'image du monde » (obraz mira) reflétée dans la langue ${ }^{47}$, ainsi que sur la « forme interne » littéraire (poétique) ${ }^{48}$ et la « forme interne » des œuvres d'art ${ }^{49}$. Gustave G. Shpet (1879-1937) lutte d'ailleurs contre le potebnisme ${ }^{50}$ qui considère finalement que le mot (slovo) comme $\lambda$ oyó (discours) contient déjà en lui les germes de la littérature : «Chaque mot, en fonction de la profondeur de notre expérience, passe nécessairement par l'état dans lequel ce mot est une création poétique $»^{51}$.

\section{Le folklore éclairé par la langue}

Potebnja s'intéresse à toutes les expressions du folklore des Ukrainiens et des Biélorusses ${ }^{52}$. Il étudie bien entendu les témoignages verbaux, notamment les chants populaires et leurs rapports avec les fêtes du calendrier et des activités humaines. Ses analyses dépassent les considérations purement formelles et il se lance même dans la description des liens entre les paroles et la musique. Il reste convaincu que la combinaison musicale 
et les sonorités verbales doivent représenter des événements archétypaux enfouis et préservés dans la mémoire collective ${ }^{53}$. Ces chants, si fréquents dans la culture ukrainienne, continuent à sustenter l'imagination nationale, forgeant ainsi une identité particulière, distincte dans l'empire de l'identité russe : le peuple ukrainien est un peuple d'élus car il a souffert et souffrira encore ${ }^{54}$. Il considère donc le folklore comme la source énergétique indispensable à toute création artistique ${ }^{55}$. Le folklore forme un réservoir inépuisable de symboles propres à une nation. Ces symboles sont diffus dans la vie de tous les jours et se combinent selon une poétique des figures et de tropes et une rhétorique des discours ${ }^{56}$.

L'un des principes de l'activité vivante des cultures consiste dans leur potentiel d'analogie: les images (obrazy) se dupliquent en plusieurs avatars qui s'adaptent en fonction du contexte de leur émission. Cela conduit Potebnja à envisager une structure absolue qui se répéterait dans chaque sphère d'activité ${ }^{57}$. Les combinaisons effectives dans les traditions orales devraient alors se retrouver dans la danse et dans les costumes. Ainsi en arrive-t-il presque à envisager toute une palette de figures psychologiques en fonction des origines de chaque coutume vestimentaire. Le costume exprime alors une histoire collective. Il constitue un " texte » et chaque détail qui le forme est un élément linguistique qui « raconte » l'histoire d'une activité dans un espace précis à une époque donnée.

Les vêtements, à l'instar de tout travail artisanal, évoluent dans le temps et l'espace, mais l'époque et le lieu en déterminent les formes et l'usage. Dès lors, il ébauche une première sémiotique de l'habillement qui confirme la particularité de la nation ukrainienne, en la distinguant de ses deux grands voisins qui sont la Russie et la Pologne $e^{58}$.

Il décrit les motifs brodés sur les chemisiers (soročki), les couleurs employées et leurs combinaisons. Il y voit des traits de caractère partagés par un état d'esprit commun, déterminés par le contexte social et géographique. La psychologie individuelle se trouve ainsi subsumée à la psychologie sociale. Il semble alors possible de définir des identités nationales, régionales, locales et mêmes familiales à partir de leurs us et coutumes.

Potebnja n'hésite pas à recourir à la psychologie et à envisager même une psychologie sociale qui intègrerait la conscience comme une "concrétisation» de notre imaginaire ${ }^{59}$. Il explique ainsi la formation du calendrier ukrainien au regard du contexte religieux et des éléments mythologiques qui s'y trouvent ${ }^{60}$.

Les articles de Potebnja consacrés au folklore ukrainiens et biélorusses sont nombreux et très dispersés. Ils sont rédigés en ukrainien, en russe et même en polonais. Aucun recueil n'a encore vu le jour. Pourtant, ils laissent entrevoir toute une théorie anthropologique de l'identité ukrainienne, liée à la terre, au travail et à la foi chrétienne ${ }^{61}$. L'étude des mythes et des légendes d'Ukraine, si bigarrés de chants et de vers, décrit l'importance des motifs religieux chrétiens et indo-européens dans l'imaginaire national. Les transformations sont nombreuses, mais une structure interne maintient toujours un noyau dur qui fait que tout Ukrainien s'adapte au milieu dans lequel il est appelé à vivre en répétant et adoptant des motifs qu'il a reçus en héritage.

La nation (narod) vient du verbe "donner naissance" (roditi). L'identité nationale relèverait de la naturalisation de la personne. La culture s'avère à ce niveau extrêmement imbriquée dans la nature ${ }^{62}$. L'appartenance nationale assure à l'individu son existence. Depuis le décès de son frère Andrej, Aleksandr œuvre en faveur de la reconnaissance de cette identité ukrainienne à la hauteur de celle des Russes et des Polonais, notamment 
dans la monographie Jazyk i narodnosড̌ (Langue et nationalité), publiée à titre posthume en $1895^{63}$. Il défend alors l'enseignement de la langue ukrainienne comme ciment indispensable pour l'identité nationale ${ }^{64}$. Cela l'amène donc à envisager un programme politique pour l'Ukraine ${ }^{65}$. L'université de Kharkiv, bien que située à quelques kilomètres de la frontière russe, a abrité un grand nombre de slavophiles soucieux d'accorder plus d'autonomie à l'Ukraine. Nikolaj Ivanovič Kostomarov (1817-1885) y est passé avant d'enseigner l'histoire à l'université de Kiev puis à celle de Saint-Pétersbourg. Il est le rédacteur en chef du fameux apocryphe intitulé Le Livre de la genèse du peuple ukrainien ${ }^{66}$ (1847) qui expose la mission messianique du peuple d'Ukraine ${ }^{67}$. Il partage avec Potebnja une véritable passion pour l'étude de la symbolique dans les traditions paléoslaves, y voyant même l'émergence d'une pensée qui caractérise chaque peuple ${ }^{68}$.

\section{En guise de conclusion}

La pensée de Potebnja témoigne d'une grande humilité. Elle n'énonce jamais une vérité sous une forme dogmatique. En revanche, elle se cache souvent derrière ou à côté des théories des grands linguistes et philosophes qu'il cite abondamment et commente. L'essence même de cette pensée réside finalement dans le commentaire et la discussion. De là découle la difficulté de la distinguer en ce qu'elle est un prolongement des idées des auteurs avec lesquels elle dialogue.

Cette manière de procéder éclaire toute la démarche épistémologique de Potebnja qui discerne une continuité entre ses travaux linguistiques, ses études folkloristes, ses critiques littéraires et son engagement politique. Il tente de mettre à jour tout un système de cohésions et de cohérences entre les idées et les formes dont est tissée la réalité. Ce projet holistique condamne sa pensée à la philosophie et soulève parfois la suspicion des spécialistes. Certes, il se fonde sur une vision foncièrement romantique des sciences qui entraîne la science vers la mystique.

Au terme de cette présentation, il est intéressant de voir qu'au-delà des récupérations idéologiques, Potebnja, même s'il n'est pas encore un penseur structuraliste ${ }^{69}$, a certainement ouvert de nombreuses voies $^{70}$. Son travail scientifique a permis à ses contemporains, Franko en tête, de fonder positivement leur volonté d'indépendance et de reconnaissance de l'Ukraine comme culture à part entière. Potebnja a sans aucun doute su décloisonner les savoirs et poser des problèmes en les éclairant sous les feux de plusieurs domaines du savoir. Il a montré que les questions politiques trouvent leur origine et leur solution non pas dans des considérations internationales mais dans l'histoire des mentalités et l'histoire des idées.

\section{NOTES}

1. ViLčkovskijJu., Oleksandr Potebnja jak filosof (Aleksandr Potebnja, philosophe), L’vivskij derzhavnyj universytet im. I. Franka, Lvov, 1995, 80 p. Voir aussi : Čiževs'kij D., Narysi z istorii filosofiï na Ukraïni (Esquisse d'histoire de la philosophie en Ukraine), Orïj pri UKSP Kobza, Kiev, 
1992, pp. 205-216 et Ogorodnik I. V. \& Ogorodnik V. V., Istorija filosofs'koï dumki v Ukraïni (Histoire de la pensée philosophique en Ukraine), Znannja, Kiev, 1999, pp. 308-312.

2. Dmitrenko N. K., A. A. Potebnja - sobirate' $i$ issledovatel' fo'klora (A. A. Potebnja, collecteur et chercheur du folklore), Znanie, Kiev, 1985, 48 p.

3. Par exemple : Skorik M., « Povernennja henija narodu» (Le Retour du génie du peuple), in : « Rozbudova derzhavi », no 9, 1996, pp. 58-60.

4. Lavrovskij P. A., Korennoe značenie $v$ nazvanijakh rodstva u slavjan (La Signification étymologique des noms de peuple chez les Slaves), Tipografia Imperatorskoj Akademii Nauk, Saint-Pétersbourg, 1867.

5. L'expression "petits Russes" désigne généralement les Ukrainiens et accessoirement les Biélorusses.

6. Strijs'kij P., «Bez ran, bez sumniviv, bez fraz... (Andrij Potebnja)» (Sans plaies, sans doutes, sans phrases... [Andrij Potebnja]), in : « PAM'JAT' STOLIT” », n 3, 2001, pp. 106-115.

7. Sljusar A., « Miž poezieju i dydaktykoju. Poetika bajki u traktuvanni O. Potebni » (Entre poésie et didactique. La poétique du conte vue par O. Potebnja), in : « SLOVo I ČAS », nº 9-10, 1995, pp. 9-13. 8. Čerems'ka O.S., «O. Potebnja ta O. Kurilo pro unormuvannja ukraïns'koï literaturnoï movi » (O. Potebnja et O. Kurilo sur la normation de la langue littéraire ukrainienne), in : Naukovi zapiski (Notes scientifiques), Ternopils'kij derzhavnij pedahohičnij universitet im. V. Hnatjuka, Ternopil', 2004, pp. 27-31.

9. Ovsjanniko-Kulikovskij D. N., «A. A. Potebnja. Potebnja kak jazykoved, myslitel’ (A. A. Potebnja. Potebnja linguiste et penseur), in : « KIEVSKAJA STARINA », $\mathrm{n}^{\circ}$ 7-9, 1893, pp. 12-20.

10. Kharciev V. I., "Osnovy poetiki A. A. Potebni » (Les Fondements de la poétique d'A. A. Potebnja), in : " VOPROSY TEORII I PSIKHOLOKHII TVORČESTVA », nº 2/2, 1910, pp. 1-2. Voir aussi : Zlenko H., «Potebnjanec' VasiL' Kharciev » (Le “Potebnianien” Vasii' Kharciev), in : « NAUkoviJ SVIT », n 1, 2006, pp. 29-30.

11. Vetukhov A. V., Jazyk, poezija i nauka (Langue, poésie et science), Kharkov, 1894 et Zagovory, zaklinanija, oberehi $i$ drugie vidy narodnago vračevanija, osnovannye na vere $v$ silu slova: iz istorii mysli (Incantations, formules de protection et autres types de guérison populaire, fondés sur la foi en le pouvoir de la parole: considérations sur l'histoire de la pensée), Tip. Var. Un. Okruga, Varsovie, 1907, $522 \mathrm{p}$.

12. Zajčenko N. I., «O. Potebnja i joho učni» (O. Potebnja et ses disciples), in: «VISNIK ČERNIHIVS'KOHO DERZHAVNOHO PEDAHOHIČNOHO UNIVERSITETU IMENI T.H. ŠEVČENKA », n ${ }^{\circ} 19,2003$, pp. 50-54.

13. Čekhovič K., O. Potebnja - ukraïn'kij mysliteL'-linhvist. Filosofija movy (O. Potebnja, un penseurlinguiste ukrainien. Philosophie du discours), Ukraïns'kij naukovij institut, Varsovie, 1931, 38 p. ; reprint in : « KHRONIKA », $\mathrm{n}^{\circ}$ 37-38, 2000, pp. 442-462.

14. Ševel’ov Ju., "Oleksandr Potebnja i ukraïns'ke pytannja » (Oleksandr Potebnja et la question ukrainienne), in: Potebnja A. A., Mova, nacionaL'nist', denacionalizacija. Statti i frahmenti (Langue, nationalité, dénationalisation. Articles et fragment ;sous la direction de Ševelov Ju.), Ukraïns'ka viL’na akademija nauku u SŠA, New-York, 1992, pp. 7-44.

15. Khluščenko V. A., "Ščob znati movu, treba znati iii istoriju: Doslidzhennja O. O. Potebni i sučasna škiL'na praktyka " (Pour connaître la langue, il faut aussi connaître l'histoire: Les recherches d'O. O. Potebnja et la pratique scolaire contemporaine), in : « VIDRoDZHENNJA ", $\mathrm{n}^{\circ} 9$, 1994, pp. 66-67.

16. Zubkova L. G., Jazyk, kuL'tura i civilizacija v koncepcii A. A. Potebni » (La Langue, la culture et la civilisation dans la conception d'A. A. Potebnja),in: « MOVA I KUL'TURA», nº 1/1, 2000, pp. 115-128. 
17. Fizer J., Alexander A. Potebnja's Psycholinguistic Theory of Literature. A metacritical inquiry (Théorie psycholinguistique de la littérature d'Alexandre A. Potebnja. Recherche métacritique), Harvard Ukrainian Research Institute, Cambridge (Mass.), 1988, 164 p.

18. Zakharova V., « Kognityvnyj aspekt metodyčnoï praktyki Oleksandra Potebni (Vykladac'ka dijaL’niš̌ linhvista)» (L'Aspect cognitif de la pratique méthodique d'Oleksandr Potebnja [Les activités pédagogiques du linguiste]), in : « RIDNA ŠKOLA », $\mathrm{n}^{\circ}$ 2, 2000, pp. 35-37. Vorobjova O.P., «Kohnityvna poetika v Potebnjans'kij retrospektyvi» (La Poétique cognitive dans la rétrospective de Potebnja), in : « MOVOZNAVSTVo », $\mathrm{n}^{\circ}$ 6, 2005, pp. 18-25.

19. Hansen-Løve O., La Révolution copernicienne du langage dans l'œuvre de Wilhelm von Humboldt, J. Vrin, Paris, 1972, 94 p.

20. Bartschat $\mathrm{Br}$., «La Réception de Humboldt dans la pensée linguistique russe, de Potebnja à Vygotskij », in : « REVUE GERMANIQUE INTERNATIONALE », $\mathrm{n}^{\circ}$ 3, 2006, pp. 13-23.

21. Steinthal H., Die Sprachwissenschaft W. von Humboldts und die Hegel'sche Philosophie (La Linguistique de W. Humboldt et la philosophie de Hegel, 1848); Klassifikation der Sprachen, dargestellt als die Entwickelung der Sprachidee (Classification des langues, exposée comme le développement de l'idée linguistique, 1850) développé sous le titre Charakteristik der hauptsächlichen Typen des Sprachbaues (Caractéristique des principaux types de construction linguistique, 1893) ; Der Ursprung der Sprache im Zusammenhang mit den Letzten Fragen Alles Wissens (L'Origine des langues au regard des dernières questions concernant la connaissance totale, 1851); Die Entwickelung der Schrift (Le Développement de l'écriture, 1852); Grammatik, Logik, Psychologie: Ihre Prinzipien und Ihre Verhältniss zu Einander (Grammaire, logique, psychologie : leurs principes et rapports mutuels, 1855) ; Geschichte der Sprachwissenschaft bei den Griechen und Römern (Histoire de la linguistique chez les Grecs et les Romains, 1863); Philologie, Geschichte und Psychologie in Ihren Gegenseitigen Beziehungen (Philologie, histoire et psychologie dans leurs relations actuelles, 1864); Die Mande-Negersprachen, Psychologisch und Phonetisch Betrachtet (La Langue des Mandes d'un point de vue psychologique et phonologique, 1867); Abriss der Sprachwissenschaft - I. Band: Einleitung in die Psychologie und Sprachwissenschaft (Aperçu de linguistique, t. 1: Introduction à la psychologie et à la linguistique, 1871); Allgemeine Ethik (Éthique générale, 1885) et $\mathrm{Zu}$ Bibel und Religionsphilosophie (Sur la Bible et la philosophie des religions, 1890).

22. Herbart J. F.: Lehrbuch der Psychologie (Manuel de psychologie, 1816); Psychologie als Wissenschaft (La Psychologie comme science, 1824-1825); Psychologische Untersuchungen (Recherches en psychologie, 1839-1840).

23. « Dans l'histoire, l'Esprit est un individu d'une nature à la fois universelle et déterminée : un peuple; et l'esprit auquel nous avons affaire est l'Esprit du Peuple. », in : Hegel G. W. F., La Raison dans l'histoire, traduit de l'allemand par Kostas Papaionnou, Union générale des éditeurs, Paris, 1983, p. 80. Consulter: Bavaresco A., La Théorie hégélienne de l'opinion publique, L'Harmattan, coll. «Ouverture philosophique », Paris, 1998, p. 80.

24. Potebnja A. A., Mova..., Op. cit. p. 63

25. Macejkiv M. A., «Psykho-linhvistyčna koncepcija tvorčosti O. Potebni » (La Conception psycholinguistique de l'œuvre d'O.Potebnja), in: Psikholohija. Zbirnik naukovykh prac' (Psychologie. Recueil de travaux scientifiques), NPU im. M.P.Drahomanova, Kiev, 2002, pp. 70-74. Zajčenko N. I., « Deščo pro fenomen kreatyvnosti u naukovij spadščini O. O. Potebni » (Sur le phénomène de la créativité dans l'héritage scientifique d'O. O. Potebnja), in : « PSIKHоLоноPEDAHOHIČNI NAUKI ", nº 3, 2003, pp. 106-110.

26. Potebnja A. A., « MysL' i jazyk », in : «SLOvo I MIR » (Sous la direction d'Arkhangel'skij A. N.), Pravda, Moscou, 1989, p. 51. 
27. Macejkiv M. A., «Pitannja psikholohiï v naukovij spadščini O. O. Potebni (Do 165-riččja z dnja narodžennja)» (Questions de psychologie dans l'héritage scientifique de O. O. Potebnja [Pour le $165^{\mathrm{e}}$ anniversaire de sa naissance]), « PEDAHOHYKA I PSYKHOLOHIJ », $\mathrm{n}^{\circ}$ 3, 2000, pp. 119-127.

28. PotebnjaA. A., « MysL' i jazyk », Art. cit., pp. 88-105.

29. Zajčenko N. I., «Filosofs'ko-pedahohyčni aspekty linhvistyčnoï koncepciï O. O. Potebni » (Aspects philo-pédagogiques de la conception linguistique de O. O. Potebnja), in : « РsүкноцоноPEDAHOHYČNI NAUKI ", n 2, 2001, pp. 141-152 et Dukhovno-cinnisni zasady pedahohičnij O. Potebni (Les Principes spirituels et axiologiques de la pédagogie d'O. Potebnja; sous la direction de Vovk L.), Nacional'nij pedahohičnij universitet im. Drahomanova, Kiev, 2006, 70 p.

30. Koyré A., La Philosophie et le problème national en Russie au début du XIXe siècle, Honoré Champion, Paris, 1929, $214 \mathrm{p}$.

31. Wakulenko S., «Wäre Alexander Potebnja ein Kantianer in der Sprachphilosophie gewesen?» (Alexandre Potebnja serait-il devenu kantien en philosophie de la langue ?), in : « STUDIA SLAVICA ", n $\mathrm{n}^{\circ}$ 50/3-4, 2005, pp. 211-233.

32. Kerecuk N., « Consciousness in Potebnja's theory of language » (La Conscience dans la théorie du langage de Potebnja), in : « HISTOIRE ÉPISTÉMOLOGIE LANGAGE », nº 22/2, 2000, pp. 81-96.

33. Potebnja A. A., « MysL' i jazyk », Art. cit., pp. 105-128.

34. Pavličenko A. N., «Razvitie lingvističeskikh idej Vil'geL'ma fon Gumboldta v naučnom tvorčestve A. A. Potebnji » (Le Développement linguistique de l'idée de Wilhelm von Humbolt dans l'œuvre scientifique de A. A. Potebnja), in: Jazyk i kus'tura: Pervaja mezhdunarodnaja konferencja (Langue et culture: première conférence internationale), Akademja, Kiev, 1992, pp. 51-52.

35. Snitko E. S., «Psikholingvističeskaja koncepcija A. A. Potebnji i sovremennaja nauka » (La Conception psycholinguistique de A. A. Potebnja et la science moderne), in : «RUSSKIJ JAZYK I LITERATURA V UČEBNYKH ZAVEDENIJAKH », $\mathrm{n}^{\circ}$ 2, 1996, pp. 3-6. Kovalevs'ka T. Ju., « Psikholinhvistična teorija O. O. Potebni v koncepcijakh syčasnoho movoznavstva » (La Théorie psycholinguistique de O. O. Potebnja dans les conceptions actuelles de l'étude du discours), in: Zapiski z zahalnoï linhvistyki. Zbirnik naukovikh prac' (Notes de linguistique générale. Recueil de travaux scientifiques), Astroprint, Odessa, 2001, pp. 74-80.

36. Leopold W., «Inner Form » (La Forme interieure), in : « LANGUAGE », 5/4, 1929, pp. 254-260.

37. Potebnja A. A., « MysL' i jazyk », Art. cit., p. 98.

38. Kis' R., Mova, dumka i kuL'turna realniš̌. Vid Oleksandra Potebni do hipotezi movnoho reljativizmu (Langue, pensée et réalité culturelle. D'Oleksandr Potebnja à l'hypothèse du relativisme langagier), Litopis, Lvov, 2002, 303 p.

39. Kokoškina E., « De Humboldt à Potebnja : évolution de la notion "d'innere Sprachform" dans la linguistique russe », in : « CAHIERS FERDINAND DE SAUSSURE », $\mathrm{n}^{\circ}$ 53, 2000, pp. 101-122.

40. Dilberman H., « Wilhelm von Humboldt et l'invention de la forme interne de la langue », in : « REVUE PHILOSOPHIQUE », $\mathrm{n}^{\circ}$ 131/2, 2006, pp. 163-191.

41. Bondarko A. V., " Pour une histoire de la notion de "contenu linguistique" (K. S. Aksakov, A. A. Potebnja)», in : « HISTOIRE ÉPISTÉMOLOGIE LANGAGE », 17/2, 1995, pp. 113-124.

42. Potebnja A. A., « MysL' i jazyk », Art. cit., p. 160.

43. Zajčenko N., «Vidobrazhennja funkcij movi u psykholoholinhvistyčnij spadščini O. O. Potebni » (Les Différentes Fonctions linguistiques dans l'héritage psycholinguistique d'O.O. Potebnja), in : « ŠLAKH OSVITI », $\mathrm{n}^{\circ}$ 1, 2002, pp. 51-54.

44. Širokorad E. Kh., «Pytannja pro vidnošennja dumki do slova v mahisters'kij disertaciï O. O. Potebni "O nekotorykh simvolakh v slavjansjoj narodnoj poezii" " (Questions concernant la reflection sur la langue dans le mémoire d'O.O. Potebnja "Sur quelques symboles dans la poésie slave populaire"), in : «MOVOZNAVSTVo ", $\mathrm{n}^{\circ}$ 6, 1995, pp. 53-59.

45. Potebnja A. A., « MysL' i jazyk », Art. cit., p. 105. 
46. Mauthner Fr., Zur Sprachwissenschaft. Beiträge zu einer Kritik der Sprache - 2. Band (Pour une linguistique. Essais d'une critique de la langue - t. 2), Böhlau, Vienne, 1999, 718 p.

47. Eismann W., Zur Geschichte des obraz-Begriffes in der russischen und sowjetischen Literaturwissenschaft (Pour une histoire du concept "d'obraz »dans la critique littéraire russe et soviétique), in: Ivanov V. V., Einführung in allgemeine Probleme der Semiotik (Introduction aux problèmes généraux de sémiotique), Wissenschaftliche Buchgesellschaft, Darmstatt, 1985, pp. 1-45.

48. Aumüller M., Innere Form und Poetizität. Die Theorie Aleksandr Potebnjas in ihrem begriffsgeschichtlichen Kontext (La Forme interne et la poéticité. La théorie d'Alexandre Potebnja dans son contexte de compréhension), Peter Lang, Francfort sur-le-Main, 2005, 284 p.

49. Berezin F. M., "W. von Humboldt en Russie et en URSS ", in : La Linguistique entre mythe et histoire. Actes des journées d'étude organisées les 4 et 5 juin 1991 à la Sorbonne en l'honneur de Hans Aarsleff (Sous la direction de Droixhe D. \& Grell Ch.), Nodus, Münster, 1993, pp. 263-273.

50. Shpet G. G., La Forme interne du mot. Études et variations sur des thèmes de Humboldt, traduit du russe par Nicolas Zavialoff, Kimé, Paris, 2007, 304 p.

51. Potebnja A. A., «Iz zapisok po teorii slovesnosti » (Des notes sur la théorie littéraire), in : Teoretičeskaja poètika (Poétique théorique; sous la direction de Muratov A. B.), Vycšaja škola, Moscou, 1990, p. 114.

52. 0. O. Potebnja i aktuaL'ni pytannja movi ta kuL'turi. Zbirnik naukovikh praci (0. O. Potebnja et les questions actuelles sur la langue et la culture.; sous la direction deAžnjuk B. M.), Dim Dmitra Burao, Kiev, 2004, 368 p.

53. Dmitrenko N. K., « Oleksandr Potebnja i narodna pisnja » (Oleksandr Potebnja et la chanson populaire), in: Ukraïn'ki narodni pisni $v$ zapisakh Oleksandra Potebni (Les Chants populaires ukrainiens dans les notes d'oleksandr Potebnja ; sous la direction de Dmitrenko N. K.), Muzična Ukraïna, Kiev, 1988, pp. 5-18.

54. Potebnja A. A., Mova..., Op. cit., p. 67.

55. Dmitrenko N. K., « Aspekti foL'kloristyčnoï koncepciï Oleksandra Potebni » (Les Aspects de la conception folkloristique d'oleksandr Potebnja), in : « SLOVO I ČAS », n 11-12, 1994, pp. 9-16.

56. Potebnja A. A., «O nekotorykh simvolakh v slavjanskoj narodnoj poezij» (Sur quelques symboles dans la poésie populaire slave), in : «SLOVo I MIR », 1867, pp. 285-378.

57. Koval'̌̌uk N. D., Doslidzhennja simvoličnykh struktur v konteksti universytets'koï nauki Ukraïni (O. Potebnja) (Recherches sur les structures symboliques dans le contexte de la science académique en Ukraine [O. Potebnja]), Institut filosofiï im. G. S. Skovorodi NAN Ukraïni, Kiev, 2007, 142 p.

58. Dmitrenko N. K., A. A. Potebnja..., Op. cit., 48 p.

59. Fontaine J., «A. A. Potebnja, figure de la linguistique russe du XIX e siècle », in : « HISTOIRE ÉPISTÉMOLOGIE LANGAGE », nº 17/2, 1995, pp. 95-111.

60. Ermolenko S. Ja., Linhvistični aspekti vzaemodii cerkovnoho ta narodnoho kalendariv i mifolohična koncepcija 0. 0. Potebni (Aspects linguistiques de l'interaction entre le calendrier de l'Église et celui du peuple), in : « MOVOZNAVSTVo », n 1, 1995, pp. 45-54.

61. Vedmedeva L., «Idei Oleksandra Potebni pro ljudynotvorču pryrodu mystectva i kuı'turnii kontekst sučasnosti ", (Les Idées d'Oleksandr Potebnja sur le caractère national de l'art) et in : Ljudynoznavči studii. Zbirnik naukovykh prac', Drohobickij derzhavnij pedahohičnij universitet im. Ivana Franka, Drohobič, 2001, pp. 63-72.

62. Karpenko Ju., «ZačynateL' ukraïns'koho narodoznavstva » (L'Initiateur de la linguistique ukrainienne), in : «FILOSOFS'KA I SOCIOLOGIČNA DUMKA », nº 1-2, 1995, pp. 23-40.

63. Zajčenko N. I., «Ukraïnoznavči interesi O. Potebni » (Les Intérêts ukrainistiques d'O. Potebnja), in: Naukovij visnik Černivec'kokho universitetu. Zbirnik naukovikh prac' (Courrier scientifique de l'Université de Černivcy. Recueil de travaux scientiques), Ruta, Černivci, 2003, pp. 74-81 et Do pitannja pro ideju narodnosti vikhovannja u tvorčij spadščini O. Potebni (Sur l'idée du 
caractère national dans l'oeuvre d'O. Potebnja), in: "VISNIK ČERNIVEC'KOHO DERZHAVNOHO PEDAHOHIČNOHO UNIVERSITETU IM. T. H. ŠEVČENKA, nº 24, 2004, pp. 113-117.

64. Kalašnik V. S., « Stavlennja O. O. Potebnja do ukraïns'koï movi ta zavdan' iï vivčennja v ocinci Jurija Ševel'ova » (La Position d'O. O. Potebnja vis-à-vis la langue ukrainienne et les tâches de son étude dans l'évaluation de Jurij Ševel'ov), in : Vidatnij filolokh sučasnosti. Naukovi vikladi na česŤ 85littja Jurija Ševel'ova, Oko, Kharkov, 1996, pp. 23-27.

65. Snitko E. S., «Jazyk i nacija: tradicii i sovremennost'. Vzgljad na jazykovuju situaciju v Ukraine s pozicij A. A. Potebni» (Langue et nation: tradition et modernité. Regard sur la situation linguistique en Ukraine du point de vue de A. A. Potebnja), in: «RUSSKIJ JAZYK I LITERATURA V UČEBNYKH ZAVEDENIJAKH », nº 1, 1996, pp. 1-3.

66. Il s'agit d'une « adaptation » inspirée des KsiĘgi narodu polskiego i pielgrzymstwa polskiego (Les Livres de la nation polonaise et du pèlerinage polonais) du poète romantique polonais Adam Mickiewicz au messianisme ukrainien.

67. Gasparov B., «La Linguistique slavophile », in : « HISTOIRE ÉPISTÉMOLOGIE LANGAGE », nº 17/2, 1995, pp. 125-145.

68. Eremenko O., «Simvolika baladi u svitli koncepcij M. I. Kostomarova ta O. O. Potebni » (La Symbolique de la ballade à la lumière des conceptions de M.I. Kostomarov et O. O. Potebnja), in : Sbornik naučnykh trudov: Literatura - Fo'klor - Problemi poetiki (Recueil de travaux scientifiques: Littérature - Folklore - Problèmes de poétique ; sous la direction de Dunaevs'ka L. F.), Tvim Inter, Kiev, 1999, pp. 42-52.

69. Aumüller M., «O vlijanii A. A. Potebni na russkykh formalistov» (Sur l'influence d'A.A. Potebnja sur les formalistes russes), in : «STUDIA SLAVICA », nº 7, 2007, pp. 193-202 ; Bya J., «Deux précurseurs du formalisme russe : Potebnja et Vesselovsky ", in :«LANGUESVIVANTES ", n³7, 1971, pp.753-765 ; Sériot P., Structure et totalité. Les origines intellectuelles du structuralisme en Europe centrale et orientale, PUF, Paris, 1999, 353 p.

70. Čobit'ko M. G., «Spadščina Potebni i rozvitok sučasnoï filolohičnoï nauki » (L'Héritage de Potebnja et l'évolution de la science philologique contemporaine), «UKRAINS'KA MOVA TA LITERATURA ", nº 3, 1998, pp. 18-25.

\section{AUTEUR}

\section{DANIEL S. LARANGÉ}

Enseignant postdoctoral à la Faculté de Lettres de l'Université McGill (Montréal), membre du CIRCÉ (Université Paris IV) et du CRIST (Université de Montréal) 